\title{
OPEN Influence of occlusal reduction on pain after endodontic treatment: a systematic review and meta-analysis
}

\begin{abstract}
Nayane Chagas Carvalho Alves, Sirley Raiane Mamede Veloso, Silmara de Andrade Silva, Andressa Cartaxo de Almeida, Christianne Tavares Velozo Telles, Kaline Romeiro, Gabriela Queiroz de Melo Monteiro $^{\bowtie}$ \& Diana Santana de Albuquerque
\end{abstract}

The purpose of this systematic review was to analyze the influence of occlusal reduction on the postoperative pain levels after endodontic treatment (instrumentation and obturation of the root canal system). This review followed the PRISMA statement and was registered at PROSPERO (CRD42018107918). Two independent reviewers searched the Lilacs, Cochrane Library, PubMed (Medline), Web of Science, Scopus, Scielo, and ScienceDirect for articles published until April 2021. The research question was, "Does occlusal reduction decrease postoperative pain in endodontically treated teeth?". Only randomized clinical trials were included. The RevMan 5 program was used for meta-analysis, calculating the relative risk (RR) and $95 \%$ confidence interval $(\mathrm{Cl})$ of the dichotomous outcome (presence or absence of pain). The search strategies retrieved 4114 studies. Twelve studies were included for qualitative analysis and nine for quantitative analysis. The meta-analysis results did not reveal a significant difference in the reduction of postoperative pain levels for endodontic instrumentation at $6,12,24,48 \mathrm{~h}$ and for endodontic obturation at 6 or $12 \mathrm{~h}$ after occlusal reduction. According to the GRADE tool, the analyzed outcome was classified as having a moderate level of certainty. It is concluded that occlusal reduction does not interfere with postoperative pain levels after endodontic treatment.

Postoperative pain results from an acute inflammatory response in periradicular tissues ${ }^{1}$ and is considered a complication of endodontic procedures with a reported incidence ranging from 3 to $58 \%{ }^{2}$. The causes of postoperative pain include inflammation in the periapical tissues ${ }^{3}$, perturbations in the endodontic microbiota, and chemical or mechanical injury caused by intracanal procedures ${ }^{1}$.

Another possible cause of pain after endodontic treatment is excessive occlusal forces in the tooth, which can trigger mechanical allodynia and extended postoperative pain ${ }^{4,5}$. Allodynia is defined as a reduction in pain threshold in response to innocuous mechanical or thermal stimulation. Preoperative mechanical allodynia in patients presenting irreversible pulpitis has been reported around $57 \%{ }^{4}$. Periradicular mechanical allodynia can contribute to the early stages of odontogenic pain because of the inflammation of vital pulpal tissue. Furthermore, a large amount of dental tissue is removed during endodontic treatment to access the root canal system, which can cause significant changes in the occlusal status ${ }^{6}$.

Hyperocclusion/occlusal trauma is a potential source of pain and fracture ${ }^{7}$. Previous studies have analyzed pain after endodontic treatment followed by an occlusal reduction (removal of all occlusal contacts) or occlusal adjustment (maintenance of normal occlusal contacts). The goal was to decrease the intensity of occlusal forces on the endodontically treated tooth and reduce the incidence of postoperative pain ${ }^{5,8-16}$. However, there is no consensus on the need for an occlusal reduction after endodontic treatment $t^{5,8,11-13,16}$.

The presence of persistent postoperative pain increased the burden on the patients who experience it and is associated with more significant healthcare ${ }^{17}$. Thus, the purpose of this systematic review was to analyze the influence of occlusal reduction on postoperative pain levels after endodontic treatment. The following hypotheses were raised: (1) occlusal reduction interferes with pain after endodontic instrumentation; (2) occlusal reduction interferes with pain after obturation of the root canal system. 


\begin{tabular}{|l|l|}
\hline Database & Search strategy \\
\hline LILACS & $(($ tw:(endodontology)) OR (tw:(endodontics))) ) AND (tw:(Postoperative Pain)) OR (tw:(Postoperative Pains)) \\
\hline Cochrane Library & $(((($ postoperative pains) OR postoperative pain) OR postoperative pain)) AND ((endodontics) OR Endodontology) \\
\hline PubMed (Medline) & $\begin{array}{l}((((\text { endodontics[MeSH Terms]) OR endodontology[Title/Abstract]) OR endodontics[Title/Abstract])) AND }(((\text { post- } \\
\text { operative pain[MeSH Terms]) OR Postoperative Pain[Title/Abstract]) OR Postoperative Pains[Title/Abstract]) }\end{array}$ \\
\hline Web of Science & TI = ("Postoperative Pain" OR "Postoperative Pains") AND TS = (Endodontics OR Endodontology) \\
\hline Scopus & $\begin{array}{l}\text { TITLE-ABS-KEY((“Postoperative Pain”) OR (“Postoperative Pain”)) AND TITLE-ABS-KEY((Endodontics) OR } \\
\text { (Endodontology)) }\end{array}$ \\
\hline Scielo & $\begin{array}{l}\text { (ti:(“Dor Pós-Operatória" OR “Dolor Posoperatorio" OR "Pain, Postoperative”)) AND (ti:((Endodontia OR Endodon- } \\
\text { cia OR Endodontics)) }\end{array}$ \\
\hline ScienceDirect & $\begin{array}{l}\text { Title, abstract or keywords((((postoperative pains) OR postoperative pain) OR postoperative pain[MeSH Terms])) } \\
\text { AND ((endodontics) OR Endodontology) }\end{array}$ \\
\hline
\end{tabular}

Table 1. Electronic databases used and search strategy. The electronic searches were performed until April 2021 with no restrictions of the start date. Studies published in English, Spanish, and Portuguese were included. $\mathrm{MeSH}$ medical subject heading.

\begin{abstract}
Materials and methods
Protocol and registration. This review was performed following the recommendations of the Cochrane Collaboration for systematic reviews ${ }^{18}$, and it was reported according to the Preferred Reporting Items for Systematic Reviews and Meta-Analyses (PRISMA) statement ${ }^{19}$. The study was registered in the PROSPERO (International Prospective Register of Systematic Reviews) database under the registration number CRD42018107918.
\end{abstract}

Eligibility criteria. The research question of this study was "Does occlusal reduction decrease postoperative pain in endodontically treated teeth?" and the PICOS of the study was then established. The population consisted of patients with teeth submitted to endodontic treatment. The intervention was an occlusal reduction (removal of all occlusal contacts) compared to occlusal adjustment (maintenance of occlusal contacts). The evaluated outcome was postoperative pain, and only clinical trials were included in the study design.

The inclusion criteria were: (1) only randomized clinical trials; (2) studies that compared occlusal reduction after endodontic treatment with a control group occlusal adjustment; (3) studies that evaluated postoperative pain. The exclusion criteria were as follows: (1) case report and series; (2) abstracts; (3) review articles; (4) in vitro studies; (5) discussions; (6) interviews; (7) editorials or opinions, and (8) clinical trials that involved patients who reported bruxism or clenching, patients treated with antibiotics or analgesics over the past $24 \mathrm{~h}$, teeth associated with swelling, presence of periodontal disease or mobility grade 1, and treatment with technical problems (e.g., root canal transportation, ledging, perforation, zipping, file fracture).

Information sources and search strategy. The databases searched were Lilacs (Latin American and Caribbean Health Sciences Literature database), Cochrane Library, PubMed (Medline), Web of Science, Scopus, Scielo, and ScienceDirect. Additionally, the reference list of the included studies was checked to identify possible relevant studies. The records were identified on the databases within a five-day interval, and the date of the last search was April 26, 2021. No software was used to retrieve searches, and manual searches were done within each database.

The search strategy was defined by performing a preliminary search using specific keywords for occlusal reduction or adjustment. However, this strategy did not retrieve relevant studies. Thus, a search was performed based on terms related to endodontic treatment and postoperative pain, in general, using "Text Words" and "Mesh Terms". The search strategies used for each database are described in Table 1.

Study selection and data collection. Two independent researchers (N.C.C.A. and A.C.A.) performed the electronic search and selected studies based on titles and abstracts that answered the research question. The duplicate removal was performed using online software (Rayyan-https:/www.rayyan.ai/) before the records were screened. After the initial search, the relevant data were extracted. All initial steps were performed independently. The following data were collected: (1) initial diagnosis; (2) type of tooth evaluated; (3) technique of chemical-mechanical preparation; (4) obturation techniques; (5) restorations; (6) intervention/comparison; (7) method of postoperative pain assessment; (8) moment of postoperative pain assessment (post obturation and post instrumentation); (9) presence of pain. Data on pain was extracted, regardless of the scale used and the type of variable (quantitative or qualitative). In case of missing information in the included articles, e-mails were sent to the corresponding authors. Contact was waited up to 15 days.

Risk of bias in individual studies. The methodological quality was examined independently by two reviewers (N.C.C.A. and A.C.A.) using the Cochrane Collaboration's tool for assessing the risk of bias. Discrepancies were resolved by a third reviewer (S.R.M.V.). In this tool, the aspects of bias risk are evaluated individually without assigning scores. They are divided into seven domains: random sequence generation, allocation concealment, blinding of participants and personnel, blinding of the outcome assessment, incomplete outcome data, selective reporting, and other sources of bias. Each domain was classified as having a low, unclear, or high risk of bias. 
Summary measures. The extracted data were analyzed using the Review Manager (RevMan) 5.3 software (The Cochrane Collaboration, Copenhagen, Denmark). The relative risk (RR) and 95\% confidence interval (CI) were calculated for each study. The data of the eligible studies were dichotomized as the presence or absence of postoperative pain.

The $\mathrm{I}^{2}$ statistic was used to evaluate the percent variation among studies due to heterogeneity, with $0-40 \%$ corresponding to might not be important heterogeneity, 30-60\% may represent moderate heterogeneity, 50-90\% may represent substantial heterogeneity, and $75-100 \%$ considerable heterogeneity ${ }^{18}$.

A sensitivity analysis was done to identify the sources of heterogeneity. However, even after removing clinical and methodological differences (Instrumentation protocol, Stage of endodontic treatment, Type of scale) from the studies from Raza et al. ${ }^{23}$; Parirokh et al. ${ }^{5}$; Emara et al. ${ }^{11}$, no significant differences were observed in the results.

The certainty of evidence assessment. According to the Grading of Recommendations, Assessment, Development, and Evaluation (GRADE) approach ${ }^{20}$, the strength of evidence was evaluated. The summary of the findings (SoF) table was constructed with the software GRADEpro GDT: GRADEpro Guideline Development Tool; McMaster University, 2015 (developed by Evidence Prime, Inc.). Each GRADE criterion was assessed individually and then computed for the certainty of the evidence. The GRADE approach classifies the certainty of evidence in one of the following four grades: high, moderate, low, or very low to achieve transparency and simplicity.

\section{Results}

Study selection. The initial search of the databases retrieved 4114 articles. After the removal of duplicates, 2987 articles remained. Titles and abstracts were read, and 13 articles were potentially eligible at this stage. After reading the full text, one article was excluded because of language (Persian) ${ }^{14}$. Finally, 12 articles ${ }^{5,9-11,13,15,16,21-24}$ were considered eligible for data extraction.

The number of patients that experienced postoperative pain varied for endodontic instrumentation. After $6 \mathrm{~h}$, the number of patients with pain varied from 15 to $115,13-87$ after $12 \mathrm{~h}, 3-72$ after $24 \mathrm{~h}$, and 6-54 patients after $48 \mathrm{~h}$. For endodontic obturation, the number of patients with pain after $6 \mathrm{~h}$ varied from 8 to 54 and 4-10 after $12 \mathrm{~h}$. Doubts arose in two studies ${ }^{11,15}$, and the authors were contacted. Only one ${ }^{11}$ provided the requested information.

Nine studies were included for quantitative synthesis ${ }^{5,9-11,15,16,21,23,24}$. The PRISMA flow diagram showing the complete selection process and inclusion of the articles is illustrated in Fig. 1.

Characteristics of the included studies. The 12 studies selected were analyzed qualitatively (Table 2). A total of 1461 endodontic treatments were performed in 1461 patients. However, this number does not comprise the data from Rosenberg et al. ${ }^{13}$ because they did not report the number of teeth submitted to endodontic treatment in the experimental groups. Sample size calculation was not reported in five studies $s^{9,10,13,16,23}$ and four studies $^{9,10,13,16}$ did not mention ethical considerations.

Included teeth had initial diagnosis [AAE/ABE, 2013] of symptomatic irreversible pulpitis ${ }^{5,9,15,16,22-24}$, symptomatic apical periodontitis ${ }^{8}$, or both symptomatic irreversible pulpitis and symptomatic apical periodontitis ${ }^{11,21}$. In the remaining two studies, the initial diagnosis was not reported ${ }^{10}$, or the diagnostic criteria were not described for the clinical trial ${ }^{13}$.

All studies included posterior teeth (premolar and molars). One study ${ }^{16}$ only evaluated premolars, and two other studies 8,24 only evaluated molars. Four studies ${ }^{9,22-24}$ mentioned including maxillary or mandibular teeth. Two studies ${ }^{11,21}$ evaluated only mandibular teeth.

For chemical-mechanical preparation, ten studies ${ }^{5,8,9,11,15,16,21-24}$ used the crown-down technique and the remaining two studies ${ }^{10,13}$ used the step-back technique. The instrumentation techniques also varied: rotary systems $^{8,11,15,21}$, reciprocating systems $s^{16,24}$, manual systems $s^{9,13,22,23}$ and one study ${ }^{10}$ did not mention the instruments used.

The use of calcium hydroxide paste ${ }^{5,9,15,22,23}$ and camphor-chlorophenol-thymol paste with dexamethasone (Endoseptone $)^{16}$ were mentioned as intracanal medication. The remaining four studies did not mention the use of any medication ${ }^{10,11,13,21}$.

The obturation techniques employed were matched single cones $^{8}$, lateral condensation ${ }^{8,11,21}$, and thermomechanical compaction ${ }^{24}$. Two studies ${ }^{8,24}$ performed the single-session obturation and used a resin epoxy-based sealer, AH plus (Dentsply Maillefer, Switzerland). However, post-obturation pain assessments used different scales for each study. Emara et al. ${ }^{11}$ and Ahmed et al. ${ }^{21}$ performed endodontic treatment in two sessions, without intracanal medication and the used Adseal (Meta, Biomed, Cheongju, South Korea), a resin epoxy-based sealer.

The extent of the occlusal surface reduction ranged from $0.5 \mathrm{~mm}^{10}, 0.5-1 \mathrm{~mm}^{13}$, and $1 \mathrm{~mm}^{5,9,15,16,22,23}$. The remaining studies did not report the extent of occlusal reduction ${ }^{8,11,21,24}$.

Pain assessment methods after endodontic treatment varied. Visual analog scale (VAS) was the most used method $^{5,8,9,11,15,21-23}$. Other methods such as verbal rating scale-VRS ${ }^{16,24}$, numerical rating scale- $\mathrm{NRS}^{24}$, and a questionnaire application ${ }^{10,13}$ were also used.

Postoperative pain was mainly assessed after instrumentation, ${ }^{5,9,10,13,15,16,22,23}$ Emara et al. ${ }^{11}$, and Ahmed et al. ${ }^{21}$ examined postoperative pain after instrumentation and obturation in two visits. Arslan et al. ${ }^{8}$ and Viana ${ }^{24}$ evaluated after obturation in a single visit. The period of pain assessment ranged from four hours after treatment ${ }^{10}$ to 7 days $^{8}$, with most studies measuring pain after 24 and $48 \mathrm{~h}^{5,9-11,15,16,21-23}$.

The restorations were performed with a resin composites ${ }^{8,11,16}$, glass ionomer ${ }^{24}$ or with a provisional sealing material based on zinc oxide $5,10,11,13,15,21,22$. Emara et al. ${ }^{11}$ and Ahmed et al. ${ }^{21}$ have reported that patients were referred to the Prosthodontics Department for final tooth restoration. Emara et al. ${ }^{11}$ even mention that at the 


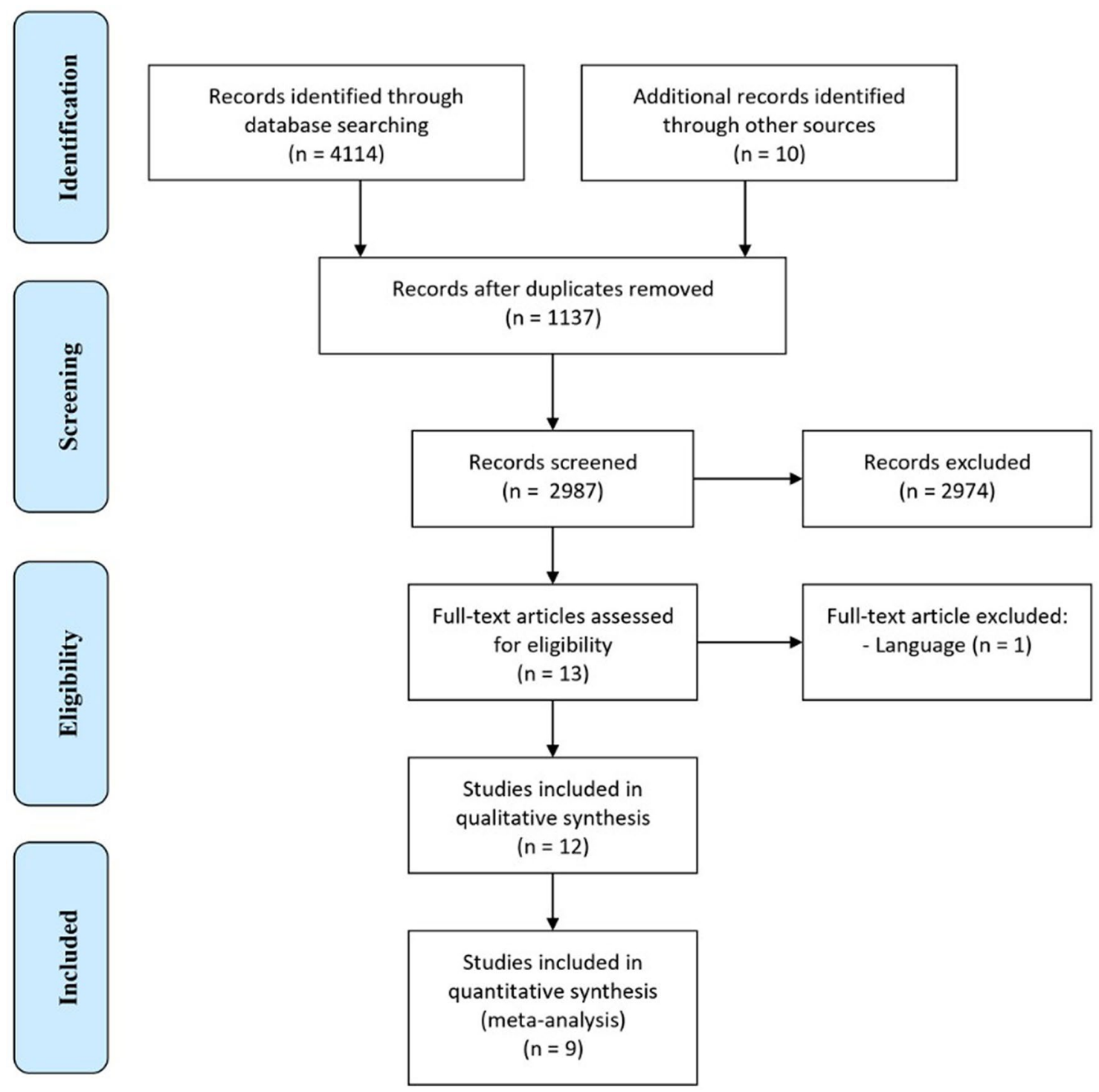

Figure 1. Preferred reporting items for systematic reviews and meta-analyses flow diagram.

end of the study and full ceramic crowns were advised for the patients in the intervention group. Asghar et al. ${ }^{9}$ and Raza et al. ${ }^{23}$ did not report the sealing material used.

The results of these primary studies are reported as the number of patients who experienced pain (presence or absence $)^{5,9-11,15,16,21,23,24}$.

Risk of bias in individual studies. The risk of bias in each study is shown in Fig. 2. Although all included studies are considered randomized clinical trials, three studies ${ }^{10,13,23}$ did not explain the random sequence generation, and four studies ${ }^{10,13,22,23}$ did not explain the method used for allocation concealment. Five studies ${ }^{5,9,13,16,23}$ did not provide information about the blinding of participants.

Blinding of outcome assessment and selective outcome reporting were classified as uncertain in only two study ${ }^{13}$. None of the studies had attrition bias due to incomplete outcome data.

All items evaluated in five studies ${ }^{8,11,15,21,24}$ were classified as low risk of bias. The risk questions answered as uncertain were related to insufficient or absent information.

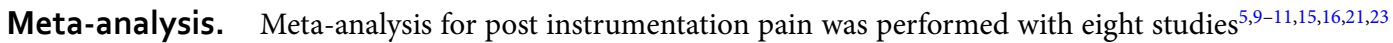
using different scales. The division into subgroups was done according to the analyzed moment (post obturation and post instrumentation). During data extraction, further subgroups were considered regarding the time interval that the measurements were performed $(6,12,24,48 \mathrm{~h})$.

Meta-analysis for post obturation of the root canal system pain was performed with three studies ${ }^{11,21,24}$ after 06 and $12 \mathrm{~h}$ using a VAS. The remaining studies differed in terms of the pain assessment scale and assessment period, which impaired the analysis. The meta-analysis was carried out using the total number of patients in 


\begin{tabular}{|c|c|c|c|c|c|c|c|c|c|c|c|c|}
\hline \multirow[b]{2}{*}{ Study } & \multirow[b]{2}{*}{ Diagnosis } & \multirow[b]{2}{*}{ Teeth } & \multicolumn{3}{|c|}{ Instrumentation protocol } & \multirow[b]{2}{*}{$\begin{array}{l}\text { Restorative } \\
\text { material }\end{array}$} & \multicolumn{3}{|c|}{ Postoperative pain assessment } & \multicolumn{2}{|l|}{ Groups (n) } & \multirow[b]{2}{*}{ Results } \\
\hline & & & Preparation & $\begin{array}{l}\mathrm{NaOCl} \\
\text { concentration } \\
(\%)\end{array}$ & $\begin{array}{l}\text { Intracanal } \\
\text { medication } \\
\text { (ICM) }\end{array}$ & & $\begin{array}{l}\text { Stage of } \\
\text { endodontic } \\
\text { treatment }\end{array}$ & $\begin{array}{l}\text { Type of } \\
\text { scale }\end{array}$ & $\begin{array}{l}\text { Experimental } \\
\text { period }\end{array}$ & \begin{tabular}{|l}
$\begin{array}{l}\text { Control } \\
\text { group } \\
\text { (occlusal } \\
\text { adjustment) }\end{array}$ \\
\end{tabular} & \begin{tabular}{|l|} 
Study \\
group \\
(occlusal \\
reduction)
\end{tabular} & \\
\hline \multirow{2}{*}{$\begin{array}{l}\text { Ahmed } \\
\text { et al. } \\
(2020)^{21}\end{array}$} & \multirow{2}{*}{$\begin{array}{l}\text { Symp- } \\
\text { tomatic } \\
\text { irreversible } \\
\text { pulpitis } \\
\text { with sen- } \\
\text { sitivity to } \\
\text { percussion }\end{array}$} & \multirow{2}{*}{$\begin{array}{l}\text { Man- } \\
\text { dibular } \\
\text { posterior } \\
\text { teeth }\end{array}$} & \multirow{2}{*}{$\begin{array}{l}\text { Crown-down } \\
\text { technique } \\
\text { Manual files } \\
\text { and Revo-S } \\
\text { rotary system }\end{array}$} & \multirow[b]{2}{*}{2.5} & \multirow{2}{*}{$\begin{array}{l}\text { No ICM } \\
\text { (sterile cot- } \\
\text { ton pellet) }\end{array}$} & $\begin{array}{l}\text { After instru- } \\
\text { mentation: } \\
\text { MD-Temp } \\
\text { (based on } \\
\text { zinc oxide) }\end{array}$ & $\begin{array}{l}\text { After instru- } \\
\text { mentation }\end{array}$ & \multirow{2}{*}{$\begin{array}{l}\text { Numeri- } \\
\text { cal rating } \\
\text { scale }\end{array}$} & $\begin{array}{l}\text { After instru- } \\
\text { mentation: } 6 \\
12,24 \text { and } 48 \mathrm{~h}\end{array}$ & \multirow{2}{*}{$\begin{array}{l}154 \text { (using } \\
\text { articulating } \\
\text { paper) }\end{array}$} & \multirow{2}{*}{$\begin{array}{l}154 \text { (not } \\
\text { report the } \\
\text { extent of } \\
\text { occlusal } \\
\text { reduction) }\end{array}$} & \multirow{2}{*}{$\begin{array}{l}\text { Significant } \\
\text { results of } \\
\text { reduction } \\
\text { in post- } \\
\text { operative } \\
\text { pain levels } \\
12 \text { and } \\
24 \mathrm{~h} \text { after } \\
\text { instru- } \\
\text { mentation }\end{array}$} \\
\hline & & & & & & \begin{tabular}{|l|} 
After obtu- \\
ration: Final \\
coronal \\
restora- \\
tion. Not \\
reported \\
\end{tabular} & $\begin{array}{l}\text { After obtu- } \\
\text { ration }\end{array}$ & & $\begin{array}{l}\text { After obtura- } \\
\text { tion: } 6 \text { and } \\
12 \mathrm{~h}\end{array}$ & & & \\
\hline $\begin{array}{l}\text { Arslan } \\
\text { et al. }^{8}\end{array}$ & \begin{tabular}{|l|} 
Symp- \\
tomatic \\
apical peri- \\
odontitis
\end{tabular} & Molars & \begin{tabular}{|l|} 
ProTaper \\
Universal \\
and K files in \\
some cases
\end{tabular} & 1.25 & - & $\begin{array}{l}\text { Fluid resin } \\
\text { and nanohy- } \\
\text { brid resin }\end{array}$ & $\begin{array}{l}\text { After obtu- } \\
\text { ration }\end{array}$ & $\begin{array}{l}\text { Visual } \\
\text { analog } \\
\text { scale }\end{array}$ & $\begin{array}{l}\text { 1st, 3rd, 5th } \\
\text { and 7th day }\end{array}$ & $\begin{array}{l}11 \text { (using a } \\
\text { computer- } \\
\text { ized analysis } \\
\text { system) }\end{array}$ & \begin{tabular}{|l}
13 (not \\
report the \\
extent of \\
occlusal \\
reduction)
\end{tabular} & $\begin{array}{l}\text { No } \\
\text { statistical } \\
\text { signifi- } \\
\text { cance }\end{array}$ \\
\hline $\begin{array}{l}\text { Asghar } \\
\text { et al. } \\
(2014)^{9}\end{array}$ & \begin{tabular}{|l|} 
Symp- \\
tomatic \\
irreversible \\
pulpitis
\end{tabular} & $\begin{array}{l}\text { Posterior } \\
\text { maxillary } \\
\text { and man- } \\
\text { dibular } \\
\text { teeth }\end{array}$ & $\begin{array}{l}\text { Gates- } \\
\text { Glidden and } \\
\text { K files }\end{array}$ & 1.3 & $\begin{array}{l}\text { Calcium } \\
\text { hydroxide } \\
\text { paste }\end{array}$ & $\begin{array}{l}\text { Provisional } \\
\text { sealing. Not } \\
\text { reported }\end{array}$ & $\begin{array}{l}\text { After instru- } \\
\text { mentation }\end{array}$ & $\begin{array}{l}\text { Visual } \\
\text { analog } \\
\text { scale }\end{array}$ & $\begin{array}{l}\text { 1st, 2nd and } \\
\text { 3rd day }\end{array}$ & $\begin{array}{l}55 \text { (using } \\
\text { articulating } \\
\text { paper) }\end{array}$ & $\begin{array}{l}55 \text { (occlusal } \\
\text { surface } \\
\text { reduced by } \\
1 \mathrm{~mm} \text { ) }\end{array}$ & $\begin{array}{l}\text { No } \\
\text { statistical } \\
\text { signifi- } \\
\text { cance }\end{array}$ \\
\hline $\begin{array}{l}\text { Creech } \\
\text { et al. } \\
(1984)^{10}\end{array}$ & $\begin{array}{l}\text { Not } \\
\text { reported }\end{array}$ & $\begin{array}{l}\text { Posterior } \\
\text { teeth }\end{array}$ & $\begin{array}{l}\text { Step-back } \\
\text { technique }\end{array}$ & 2.5 & $\begin{array}{l}\text { No ICM } \\
\text { (sterile cot- } \\
\text { ton pellet) }\end{array}$ & $\begin{array}{l}\text { Provisional } \\
\text { sealing: } \\
\text { Cavit (based } \\
\text { on zinc } \\
\text { oxide) }\end{array}$ & $\begin{array}{l}\text { After instru- } \\
\text { mentation }\end{array}$ & $\begin{array}{l}\text { Question- } \\
\text { naire }\end{array}$ & $\begin{array}{l}4,8,24,36 \text { and } \\
48 \mathrm{~h}\end{array}$ & $\begin{array}{l}25 \text { (using } \\
\text { articulating } \\
\text { paper) }\end{array}$ & $\begin{array}{l}24 \text { (occlusal } \\
\text { surface } \\
\text { reduced by } \\
0.5 \mathrm{~mm} \text { ) }\end{array}$ & $\begin{array}{l}\text { No } \\
\text { statistical } \\
\text { signifi- } \\
\text { cance }\end{array}$ \\
\hline \multirow{2}{*}{$\begin{array}{l}\text { Emara } \\
\text { et al. } \\
(2019)^{11}\end{array}$} & \multirow{2}{*}{$\begin{array}{l}\text { Symp- } \\
\text { tomatic } \\
\text { irreversible } \\
\text { pulpitis; } \\
\text { symp- } \\
\text { tomatic } \\
\text { apical peri- } \\
\text { odontitis }\end{array}$} & \multirow{2}{*}{$\begin{array}{l}\text { Man- } \\
\text { dibular } \\
\text { posterior } \\
\text { teeth }\end{array}$} & \multirow{2}{*}{$\begin{array}{l}\text { Crown-down } \\
\text { technique } \\
\text { Manual files } \\
\text { and Revo-S } \\
\text { rotary system }\end{array}$} & \multirow{2}{*}{2.5} & \multirow{2}{*}{$\begin{array}{l}\text { No ICM } \\
\text { (sterile cot- } \\
\text { ton pellet) }\end{array}$} & $\begin{array}{l}\text { After instru- } \\
\text { mentation: } \\
\text { MD-Temp } \\
\text { (based on } \\
\text { zinc oxide) }\end{array}$ & $\begin{array}{l}\text { After instru- } \\
\text { mentation }\end{array}$ & \multirow{2}{*}{$\begin{array}{l}\text { Visual } \\
\text { analog } \\
\text { scale }\end{array}$} & \begin{tabular}{|l|} 
After instru- \\
mentation: 6 \\
12,24 and $48 \mathrm{~h}$
\end{tabular} & \multirow{2}{*}{$\begin{array}{l}22 \text { (using } \\
\text { articulating } \\
\text { paper) }\end{array}$} & \multirow{2}{*}{$\begin{array}{l}22 \text { (not } \\
\text { report the } \\
\text { extent of } \\
\text { occlusal } \\
\text { reduction) }\end{array}$} & \multirow{2}{*}{\begin{tabular}{|l|} 
Significant \\
results of \\
reduction \\
in post- \\
operative \\
pain levels \\
$12 \mathrm{~h}$ after \\
instru- \\
mentation \\
and obtu- \\
ration \\
\end{tabular}} \\
\hline & & & & & & $\begin{array}{l}\text { After } \\
\text { obturation: } \\
\text { Resin and/ } \\
\text { or ceramic } \\
\text { crown }\end{array}$ & $\begin{array}{l}\text { After obtu- } \\
\text { ration }\end{array}$ & & $\begin{array}{l}\text { After obtura- } \\
\text { tion: } 6 \text { and } \\
12 \mathrm{~h}\end{array}$ & & & \\
\hline $\begin{array}{l}\text { Parirokh } \\
\text { et al. } \\
(2013)^{5}\end{array}$ & \begin{tabular}{|l|} 
Symp- \\
tomatic \\
irreversible \\
pulpitis
\end{tabular} & $\begin{array}{l}\text { Premo- } \\
\text { lar and } \\
\text { molars }\end{array}$ & \begin{tabular}{|l|} 
Manual files, \\
Gates-Glid- \\
den drills \\
and HERO \\
642 rotary \\
instruments
\end{tabular} & 1.3 & $\begin{array}{l}\text { Calcium } \\
\text { hydroxide } \\
\text { paste }\end{array}$ & $\begin{array}{l}\text { Provisional } \\
\text { sealing: } \\
\text { Cotosol } \\
\text { (based on } \\
\text { zinc oxide) }\end{array}$ & $\begin{array}{l}\text { After instru- } \\
\text { mentation }\end{array}$ & $\begin{array}{l}\text { Visual } \\
\text { analog } \\
\text { scale }\end{array}$ & $\begin{array}{l}6,12,18,24 \mathrm{~h} \\
\text {,2nd, 3rd, 4th, } \\
\text { 5th and 6th } \\
\text { day }\end{array}$ & $\begin{array}{l}21 \text { (using } \\
\text { articulating } \\
\text { paper) }\end{array}$ & $\begin{array}{l}25 \text { (occlusal } \\
\text { surface } \\
\text { reduced by } \\
1 \mathrm{~mm} \text { ) }\end{array}$ & $\begin{array}{l}\text { No } \\
\text { statistical } \\
\text { signifi- } \\
\text { cance }\end{array}$ \\
\hline $\begin{array}{l}\text { Raza et al. } \\
(2016)^{23}\end{array}$ & \begin{tabular}{|l|} 
Symp- \\
tomatic \\
irreversible \\
pulpitis
\end{tabular} & $\begin{array}{l}\text { posterior } \\
\text { maxillary } \\
\text { and man- } \\
\text { dibular } \\
\text { teeth }\end{array}$ & $\begin{array}{l}\text { Gates- } \\
\text { Glidden and } \\
\text { K files }\end{array}$ & 1.3 & $\begin{array}{l}\text { Calcium } \\
\text { hydroxide } \\
\text { paste }\end{array}$ & $\begin{array}{l}\text { Provisional } \\
\text { sealing. Not } \\
\text { reported }\end{array}$ & $\begin{array}{l}\text { After instru- } \\
\text { mentation }\end{array}$ & $\begin{array}{l}\text { Visual } \\
\text { analog } \\
\text { scale }\end{array}$ & $24 \mathrm{~h}$ & $\begin{array}{l}55 \text { (using } \\
\text { articulating } \\
\text { paper) }\end{array}$ & $\begin{array}{l}55 \text { (occlusal } \\
\text { surface } \\
\text { reduced by } \\
1 \mathrm{~mm} \text { ) }\end{array}$ & $\begin{array}{l}\text { No } \\
\text { statistical } \\
\text { signifi- } \\
\text { cance }\end{array}$ \\
\hline $\begin{array}{l}\text { Rosen- } \\
\text { berg et al. } \\
(1998)^{13}\end{array}$ & $\begin{array}{l}\text { Without } \\
\text { specifica- } \\
\text { tions }\end{array}$ & $\begin{array}{l}\text { Posterior } \\
\text { teeth }\end{array}$ & $\begin{array}{l}\text { Step-back } \\
\text { technique } \\
\text { Manual files }\end{array}$ & 2 & $\begin{array}{l}\text { No ICM } \\
\text { (sterile cot- } \\
\text { ton pellet) }\end{array}$ & $\begin{array}{l}\text { Provisional } \\
\text { sealing: } \\
\text { Cavit (based } \\
\text { on zinc } \\
\text { oxide) }\end{array}$ & $\begin{array}{l}\text { After instru- } \\
\text { mentation }\end{array}$ & $\begin{array}{l}\text { Question- } \\
\text { naire }\end{array}$ & Over $48 \mathrm{~h}$ & $\begin{array}{l}\text { Not reported } \\
\text { (using } \\
\text { articulating } \\
\text { paper) }\end{array}$ & $\begin{array}{l}\text { Not } \\
\text { reported } \\
\text { (occlusal } \\
\text { surface } \\
\text { reduced } \\
\text { by } 0.5 \text { to } \\
1.0 \mathrm{~mm} \text { ) }\end{array}$ & $\begin{array}{l}\text { Occlusal } \\
\text { reduction } \\
\text { aids in the } \\
\text { preven- } \\
\text { tion of } \\
\text { postopera- } \\
\text { tive pain } \\
\text { in teeth } \\
\text { with vital } \\
\text { pulp, } \\
\text { percussion } \\
\text { sensitivity, } \\
\text { preopera- } \\
\text { tive pain, } \\
\text { and/or } \\
\text { absence of } \\
\text { per- } \\
\text { iradicular } \\
\text { radiolu- } \\
\text { cency } \\
\end{array}$ \\
\hline $\begin{array}{l}\text { Sheikh } \\
\text { et al. } \\
(2015)^{15}\end{array}$ & \begin{tabular}{|l|} 
Symp- \\
tomatic \\
irreversible \\
pulpitis
\end{tabular} & $\begin{array}{l}\text { Posterior } \\
\text { teeth }\end{array}$ & \begin{tabular}{|l|} 
Manual \\
instruments, \\
Gates- \\
Glidden \\
drills and \\
ProTaper F1 \\
or F2 rotary \\
instruments
\end{tabular} & 3 & $\begin{array}{l}\text { Calcium } \\
\text { hydroxide } \\
\text { paste }\end{array}$ & $\begin{array}{l}\text { Provisional } \\
\text { sealing: } \\
\text { Cavit (based } \\
\text { on zinc } \\
\text { oxide) }\end{array}$ & $\begin{array}{l}\text { After instru- } \\
\text { mentation }\end{array}$ & $\begin{array}{l}\text { Visual } \\
\text { analog } \\
\text { scale }\end{array}$ & $\begin{array}{l}\text { 6, 12, 18, 24 h, } \\
\text { 2nd, 3rd, 4th, } \\
\text { 5th and 6th } \\
\text { day }\end{array}$ & $\begin{array}{l}201 \text { (using } \\
\text { articulating } \\
\text { paper) }\end{array}$ & $\begin{array}{l}201 \\
\text { (occlusal } \\
\text { surface } \\
\text { reduced by } \\
1 \mathrm{~mm} \text { ) }\end{array}$ & $\begin{array}{l}\text { The mean } \\
\text { pain } \\
\text { score was } \\
\text { significant } \\
6 \text { days } \\
\text { after } \\
\text { instru- } \\
\text { mentation }\end{array}$ \\
\hline
\end{tabular}




\begin{tabular}{|c|c|c|c|c|c|c|c|c|c|c|c|c|}
\hline \multirow[b]{2}{*}{ Study } & \multirow[b]{2}{*}{ Diagnosis } & \multirow[b]{2}{*}{ Teeth } & \multicolumn{3}{|c|}{ Instrumentation protocol } & \multirow[b]{2}{*}{$\begin{array}{l}\text { Restorative } \\
\text { material }\end{array}$} & \multicolumn{3}{|c|}{ Postoperative pain assessment } & \multicolumn{2}{|l|}{ Groups (n) } & \multirow[b]{2}{*}{ Results } \\
\hline & & & Preparation & $\begin{array}{l}\mathrm{NaOCl} \\
\text { concentration } \\
(\%)\end{array}$ & $\begin{array}{l}\text { Intracanal } \\
\text { medication } \\
(\text { ICM) }\end{array}$ & & $\begin{array}{l}\text { Stage of } \\
\text { endodontic } \\
\text { treatment }\end{array}$ & $\begin{array}{l}\text { Type of } \\
\text { scale }\end{array}$ & $\begin{array}{l}\text { Experimental } \\
\text { period }\end{array}$ & $\begin{array}{l}\text { Control } \\
\text { group } \\
\text { (occlusal } \\
\text { adjustment) }\end{array}$ & \begin{tabular}{|l|} 
Study \\
group \\
(occlusal \\
reduction)
\end{tabular} & \\
\hline $\begin{array}{l}\text { Viana } \\
\text { et al. } \\
(2020)^{24}\end{array}$ & $\begin{array}{l}\text { Symp- } \\
\text { tomatic } \\
\text { irreversible } \\
\text { pulpitis }\end{array}$ & $\begin{array}{l}\text { Maxillary } \\
\text { and man- } \\
\text { dibular } \\
\text { molars }\end{array}$ & $\begin{array}{l}\text { Proglider } \\
\text { and } \\
\text { WaveOne } \\
\text { Gold systems }\end{array}$ & 2.5 & - & $\begin{array}{l}\text { Provisional } \\
\text { sealing: } \\
\text { Glass iono- } \\
\text { mer }\end{array}$ & $\begin{array}{l}\text { After obtu- } \\
\text { ration }\end{array}$ & $\begin{array}{l}\text { Verbal } \\
\text { rating } \\
\text { scale and } \\
\text { numeri- } \\
\text { cal rating } \\
\text { scale }\end{array}$ & 6,24 and $72 \mathrm{~h}$ & $\begin{array}{l}40 \text { (using } \\
\text { articulating } \\
\text { paper) }\end{array}$ & $\begin{array}{l}38 \text { (not } \\
\text { report the } \\
\text { extent of } \\
\text { occlusal } \\
\text { reduction) }\end{array}$ & $\begin{array}{l}\text { No } \\
\text { statistical } \\
\text { signifi- } \\
\text { cance }\end{array}$ \\
\hline $\begin{array}{l}\text { Zaman } \\
\text { and } \\
\text { Ahmed } \\
(2016)^{22}\end{array}$ & $\begin{array}{l}\text { Symp- } \\
\text { tomatic } \\
\text { irreversible } \\
\text { pulpitis }\end{array}$ & $\begin{array}{l}\text { posterior } \\
\text { maxillary } \\
\text { and man- } \\
\text { dibular } \\
\text { teeth }\end{array}$ & $\begin{array}{l}\text { Gates- } \\
\text { Glidden and } \\
\text { K files }\end{array}$ & 2.5 & $\begin{array}{l}\text { Calcium } \\
\text { hydroxide } \\
\text { paste }\end{array}$ & $\begin{array}{l}\text { Provisional } \\
\text { sealing: } \\
\text { Cavit (based } \\
\text { on zinc } \\
\text { oxide) }\end{array}$ & $\begin{array}{l}\text { After instru- } \\
\text { mentation }\end{array}$ & $\begin{array}{l}\text { Visual } \\
\text { analog } \\
\text { scale }\end{array}$ & $\begin{array}{l}24 \mathrm{~h}, 2 \mathrm{nd}, 3 \mathrm{rd}, \\
4 \text { th, 5th and } \\
6 \text { th day }\end{array}$ & $\begin{array}{l}125 \text { (using } \\
\text { articulating } \\
\text { paper) }\end{array}$ & $\begin{array}{l}125 \\
\text { (occlusal } \\
\text { surface } \\
\text { reduced by } \\
1 \mathrm{~mm} \text { ) }\end{array}$ & $\begin{array}{l}\text { The mean } \\
\text { pain } \\
\text { score was } \\
\text { significant } \\
6 \text { days } \\
\text { after } \\
\text { instru- } \\
\text { mentation }\end{array}$ \\
\hline $\begin{array}{l}\text { Zeidan } \\
(2016)^{16}\end{array}$ & $\begin{array}{l}\text { Symp- } \\
\text { tomatic } \\
\text { irreversible } \\
\text { pulpitis }\end{array}$ & $\begin{array}{l}\text { Premo- } \\
\text { lars }\end{array}$ & $\begin{array}{l}\text { Manual } \\
\text { instru- } \\
\text { ments and } \\
\text { WaveOne } \\
\text { Primary }\end{array}$ & 2 & $\begin{array}{l}\text { Endosep- } \\
\text { tone }\end{array}$ & $\begin{array}{l}\text { Resin-rein- } \\
\text { forced glass } \\
\text { ionomer }\end{array}$ & $\begin{array}{l}\text { After instru- } \\
\text { mentation }\end{array}$ & $\begin{array}{l}\text { Verbal } \\
\text { rating } \\
\text { scale }\end{array}$ & 12,24 and $48 \mathrm{~h}$ & $\begin{array}{l}20 \text { (using } \\
\text { articulating } \\
\text { paper) }\end{array}$ & $\begin{array}{l}20 \text { (occlusal } \\
\text { surface } \\
\text { reduced by } \\
1 \mathrm{~mm})\end{array}$ & $\begin{array}{l}\text { No } \\
\text { statistical } \\
\text { signifi- } \\
\text { cance }\end{array}$ \\
\hline
\end{tabular}

Table 2. Evidence table summarizing the characteristics of the included studies.

each experimental group and the number of patients with pain, irrespective of its intensity. Forest plots of comparison were constructed.

Meta-analysis for post instrumentation pain did not reveal a significant difference in the reduction of postoperative pain levels after occlusal reduction after $6 \mathrm{~h}\left(\mathrm{p}=0.71\right.$; RR: $\left.0.96 ; 95 \% \mathrm{CI} 0.77-1.19 ; \mathrm{I}^{2}=50 \% ; \mathrm{p}=0.13\right)$, $12 \mathrm{~h}\left(\mathrm{p}=0.09\right.$; RR: 0.86; 95\% CI 0.72-1.02; $\left.\mathrm{I}^{2}=11 \% ; \mathrm{p}=0.34\right), 24 \mathrm{~h}\left(\mathrm{p}=0.39\right.$; RR: $0.91 ; 95 \%$ CI $0.73-1.13 ; \mathrm{I}^{2}=30 \%$; $\mathrm{p}=0.20)$ and $48 \mathrm{~h}\left(\mathrm{p}=0.57\right.$; RR: $0.94 ; 95 \%$ CI $\left.0.74-1.18 ; \mathrm{I}^{2}=0 \% ; \mathrm{p}=0.98\right)$. Meta-analysis for post obturation of the root canal system, did not reveal a significant difference in the reduction of postoperative pain levels after occlusal reduction after $6 \mathrm{~h}\left(\mathrm{p}=0.32\right.$; RR: $0.89 ; 95 \%$ CI $\left.0.72-1.11 ; \mathrm{I}^{2}=0 \% ; \mathrm{p}=0.49\right)$ and after $12 \mathrm{~h}(\mathrm{p}=0.33$; RR: $0.80 ; 95 \%$ CI $\left.0.51-1.25 ; \mathrm{I}^{2}=53 \% ; \mathrm{p}=0.12\right)$.

Certainty of evidence. The quality of evidence and the strength of recommendation of the main outcomes evaluated by the GRADE tool were rated as moderate. According to GRADE's definition of moderate, "The true effect is likely to be close to the estimate of the effect, but there is a possibility that it is substantially different". However, due to categorization, some degree of arbitrariness can be inputted. There is a possibility that the true effect and the effect's estimate are substantially different (Table 3).

\section{Discussion}

The results of this systematic review show that occlusal reduction does not interfere with pain after the endodontic treatment in the first $48 \mathrm{~h}$. Postoperative pain after endodontic treatment was separately evaluated after instrumentation and after obturation. The hypothesis that occlusal reduction interferes with pain after endodontic instrumentation and obturation was rejected. The meta-analysis did not favor either studied clinical approach. This systematic review included only clinical trials. However, many factors can influence the incidence of postoperative pain: the diagnosis and classification of the included teeth, the instrumentation techniques, the determination of working length, the type of irrigating solution, the use of intracanal medication, and obturation techniques.

Overall, five studies ${ }^{11,13,15,21,22}$ demonstrated significant results of occlusal reduction in preventing postoperative pain after instrumentation. However, in three of these five studies, the experimental period was the sum of pain incidence over $48 \mathrm{~h}^{13}$ or up to 6 days ${ }^{15,22}$. Postoperative pain should not be summed over experimental time points since it does not correspond to the incidence during a given period. In this respect, only Emara et al. ${ }^{11}$ and Ahmed et al. ${ }^{21}$ were included in the meta-analysis. They demonstrated a decrease in postoperative pain $12 \mathrm{~h}$ after occlusal reduction for both chemical-mechanical preparation and obturation.

The presence of preoperative pain can also influence the presence of postoperative pain ${ }^{25,26}$. Most of the meta-

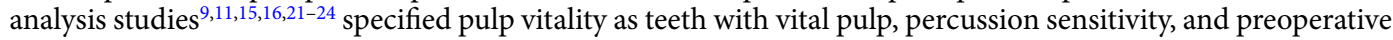
pain. Postoperative pain is significantly associated with previous painful symptoms in teeth without periradicular lesions, probably due to the lack of space for pressure release during instrumentation ${ }^{27}$ However, Alí et al. ${ }^{26}$ reported that pulp vitality does not affect postoperative pain intensity or frequency. Only two studies ${ }^{8,11} \mathrm{empha}^{-}$ sized the periapical diagnosis, including teeth with symptomatic apical periodontitis. Therefore, symptomatic irreversible pulpitis diagnosis may not interfere with pain after endodontic treatment with occlusal reduction.

A higher incidence of pain after endodontic treatment has been reported in molars. A higher number of canals can favor periapical pain ${ }^{25,28}$. In this review, most of the evaluated studies ${ }^{21-23}$ included molars and premolars, favoring the occurrence of postoperative pain in control groups (maintenance of normal occlusal contacts) and less pain in the intervention group (occlusal reduction). However, this meta-analyzes showed no significant differences. 


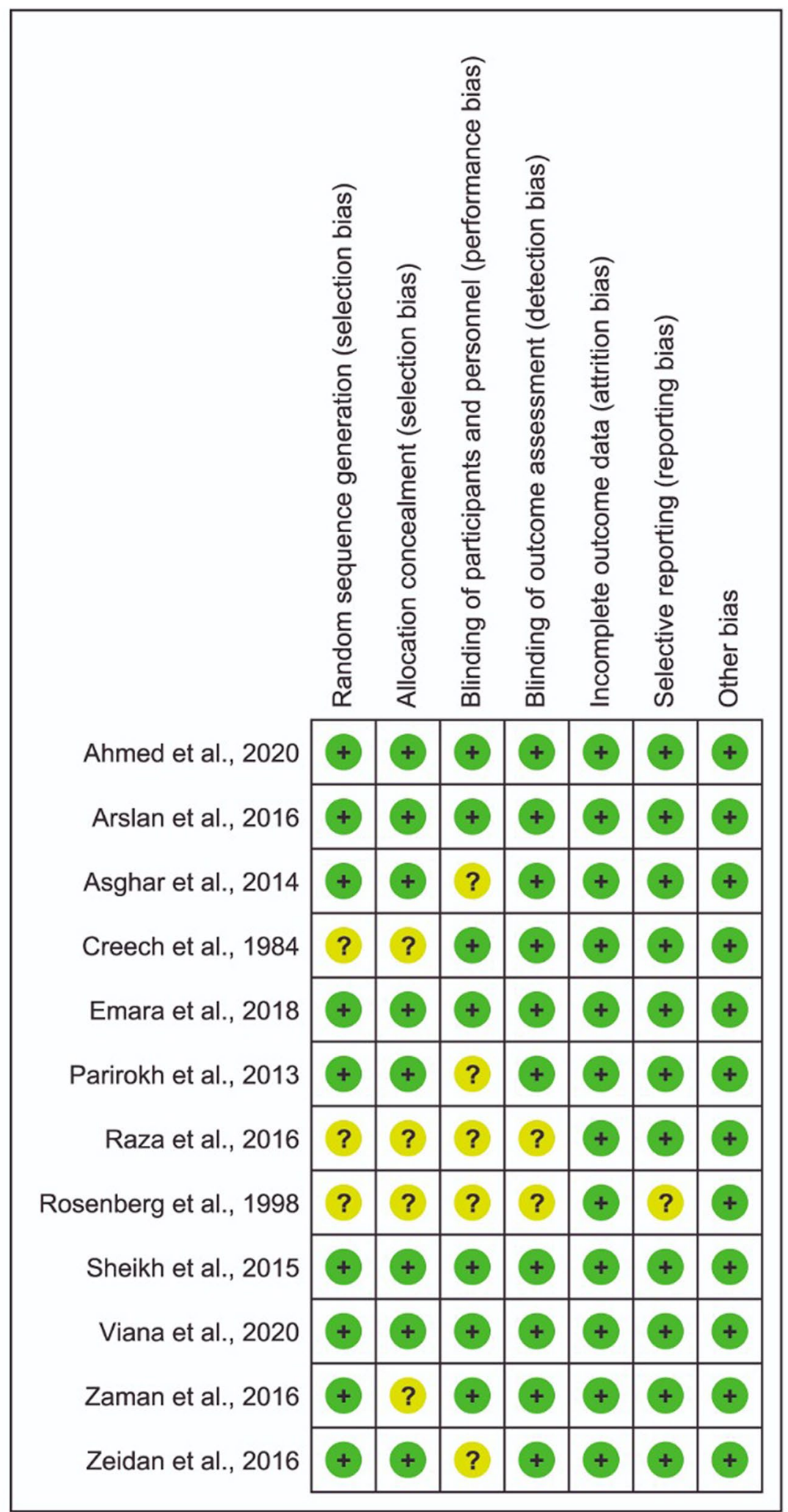

Figure 2. Risk of bias assessment of the included studies. 


\begin{tabular}{|c|c|c|c|c|c|c|c|c|c|c|c|c|}
\hline \multicolumn{7}{|c|}{ Certainty assessment } & \multicolumn{2}{|c|}{ No. of patients } & \multicolumn{2}{|l|}{ Effect } & \multirow{2}{*}{\begin{tabular}{|l|} 
Result \\
Risk ratio \\
M-H, \\
random, \\
95\% CI \\
\end{tabular}} & \multirow[b]{2}{*}{ Certainty } \\
\hline $\begin{array}{l}\text { No. of } \\
\text { studies }\end{array}$ & $\begin{array}{l}\text { Study } \\
\text { design }\end{array}$ & $\begin{array}{l}\text { Risk of } \\
\text { bias }\end{array}$ & Inconsistency & Indirectness & Imprecision & $\begin{array}{l}\text { Other } \\
\text { considerations }\end{array}$ & $\begin{array}{l}\text { Occlusal } \\
\text { reduction }\end{array}$ & $\begin{array}{l}\text { Occlusal } \\
\text { adjustment }\end{array}$ & $\begin{array}{l}\text { Relative } \\
(95 \% \text { CI })\end{array}$ & $\begin{array}{l}\text { Absolute } \\
\text { (95\% CI) }\end{array}$ & & \\
\hline 3 & $\begin{array}{l}\text { Ran- } \\
\text { domised } \\
\text { trials }\end{array}$ & $\begin{array}{l}\text { Not seri- } \\
\text { ous }\end{array}$ & Not serious & Not serious & Serious $^{\mathrm{a}}$ & None & $\begin{array}{l}149 / 201 \\
(74.1 \%)\end{array}$ & $\begin{array}{l}149 / 197 \\
(75.6 \%)\end{array}$ & $\begin{array}{l}\text { RR } 0.96 \\
(0.77- \\
1.19)\end{array}$ & \begin{tabular}{|l|}
30 fewer \\
per 1000 \\
(from 174 \\
fewer to \\
144 more) \\
\end{tabular} & $\begin{array}{l}0.96[0.77, \\
1.19]\end{array}$ & $\begin{array}{l}\oplus \oplus \oplus \bigcirc \\
\text { MODER- } \\
\text { ATE }\end{array}$ \\
\hline 4 & $\begin{array}{l}\text { Ran- } \\
\text { domised } \\
\text { trials }\end{array}$ & $\begin{array}{l}\text { Not seri- } \\
\text { ous }\end{array}$ & Not serious & Not serious & Serious $^{\mathrm{a}}$ & None & $\begin{array}{l}131 / 241 \\
(54.4 \%)\end{array}$ & $\begin{array}{l}148 / 237 \\
(62.4 \%)\end{array}$ & $\begin{array}{l}\text { RR } 0.86 \\
(0.72- \\
1.02)\end{array}$ & \begin{tabular}{|l|}
87 fewer \\
per 1000 \\
(from 175 \\
fewer to 12 \\
more)
\end{tabular} & $\begin{array}{l}0.86[0.72, \\
1.02]\end{array}$ & $\begin{array}{l}\oplus \oplus \oplus \bigcirc \\
\text { MODER- } \\
\text { ATE }\end{array}$ \\
\hline 7 & $\begin{array}{l}\text { Ran- } \\
\text { domised } \\
\text { trials }\end{array}$ & $\begin{array}{l}\text { Not seri- } \\
\text { ous }\end{array}$ & Not serious & Not serious & Serious $^{\mathrm{a}}$ & NONE & $\begin{array}{l}147 / 370 \\
(39.7 \%)\end{array}$ & $\begin{array}{l}173 / 367 \\
(47.1 \%)\end{array}$ & $\begin{array}{l}\text { RR 0.91 } \\
(0.73- \\
1.13)\end{array}$ & $\begin{array}{l}42 \text { fewer } \\
\text { per } 1000 \\
\text { (from } 127 \\
\text { fewer to } 61 \\
\text { more) }\end{array}$ & $\begin{array}{l}0.91[0.73, \\
1.13]\end{array}$ & $\begin{array}{l}\oplus \oplus \oplus \bigcirc \\
\text { MODER- } \\
\text { ATE }\end{array}$ \\
\hline 6 & $\begin{array}{l}\text { Ran- } \\
\text { domised } \\
\text { trials }\end{array}$ & $\begin{array}{l}\text { Not seri- } \\
\text { ous }\end{array}$ & Not serious & Not serious & Serious $^{\mathrm{a}}$ & None & $\begin{array}{l}95 / 320 \\
(29.7 \%)\end{array}$ & $\begin{array}{l}101 / 317 \\
(31.9 \%)\end{array}$ & $\begin{array}{l}\text { RR } 0.94 \\
(0.74 \text { to } \\
1.18)\end{array}$ & $\begin{array}{l}19 \text { fewer } \\
\text { per } 1000 \\
\text { (from } 83 \\
\text { fewer to } 57 \\
\text { more) }\end{array}$ & $\begin{array}{l}0.94[0.74, \\
1.18]\end{array}$ & $\begin{array}{l}\oplus \oplus \oplus \bigcirc \\
\text { MODER- } \\
\text { ATE }\end{array}$ \\
\hline 3 & $\begin{array}{l}\text { Ran- } \\
\text { domised } \\
\text { trials }\end{array}$ & $\begin{array}{l}\text { Not seri- } \\
\text { ous }\end{array}$ & Not serious & Not serious & Serious $^{\mathrm{a}}$ & None & $\begin{array}{l}83 / 214 \\
(38.8 \%)\end{array}$ & $\begin{array}{l}94 / 216 \\
(43.5 \%)\end{array}$ & $\begin{array}{l}\text { RR } 0.89 \\
(0.72 \text { to } \\
1.11)\end{array}$ & \begin{tabular}{|l}
48 fewer \\
per 1000 \\
(from 122 \\
fewer to 48 \\
more) \\
\end{tabular} & $\begin{array}{l}0.89[0.72, \\
1.11]\end{array}$ & $\begin{array}{l}\oplus \oplus \oplus \bigcirc \\
\text { MODER- } \\
\text { ATE }\end{array}$ \\
\hline 3 & $\begin{array}{l}\text { Ran- } \\
\text { domised } \\
\text { trials }\end{array}$ & $\begin{array}{l}\text { Not seri- } \\
\text { ous }\end{array}$ & Not serious & Not serious & Serious $^{\mathrm{a}}$ & None & $\begin{array}{l}62 / 214 \\
(29.0 \%)\end{array}$ & $\begin{array}{l}75 / 216 \\
(34.7 \%)\end{array}$ & $\begin{array}{l}\text { RR } 0.80 \\
(0.51 \text { to } \\
1.25)\end{array}$ & $\begin{array}{l}69 \text { fewer } \\
\text { per } 1000 \\
\text { (from } 170 \\
\text { fewer to } 87 \\
\text { more) }\end{array}$ & $\begin{array}{l}0.80[0.51 \\
1.25]\end{array}$ & $\begin{array}{l}\oplus \oplus \oplus \bigcirc \\
\text { MODER- } \\
\text { ATE }\end{array}$ \\
\hline
\end{tabular}

Table 3. Grade of Recommendation, Assessment, Development, and Evaluation (GRADE) based on the characteristics of studies included in the systematic review and meta-analysis. GRADE approach results in an assessment of the quality of a body of evidence High: Very confident that the true effect lies close to that of the estimate of the effect. Moderate: Moderately confident in the effect estimate, the true effect is likely to be close to the estimate of the effect, but there is a possibility that it is substantially different. Low: Limited confidence in the effect estimate, the true effect may be substantially different from the estimate of the effect. Very low: Little confidence in the effect estimate, the true effect is likely to be substantially different from the estimate of effect. ${ }^{a}$ The confidence interval (CI) cross the clinical decision threshold between recommending and not recommending treatment.

The instrumentation techniques used were rotary systems in five studies ${ }^{5,8,11,15,21}$ reciprocating systems in two ${ }^{16,24}$, and manual systems in five other studies ${ }^{9,10,13,22,23}$ Instrumentation techniques as the modified step-back, reciprocating, and rotary systems have been shown to cause postoperative pain ${ }^{29}$. Mechanical instruments such as continuous rotary and reciprocating systems are equivalent in terms of postoperative pain ${ }^{30,31}$. Few studies in the literature compare manual preparation techniques and automated systems ${ }^{32}$. Previous systematic reviews showed that rotary instruments' use contributed to a lower incidence and intensity of postoperative pain than manual files after single-visit root canal treatment. The use of multiple rotary-file systems contributed to a lower incidence of postoperative pain than reciprocating systems ${ }^{32}$. In the present review, three studies ${ }^{11,15,21}$ that performed rotary systems significantly reduced postoperative pain after occlusal reduction, although metanalysis did not reveal significant differences.

Concerning the irrigating solution, sodium hypochlorite (1.25-3\%) was the irrigant solution in all selected studies. There is no consensus on the optimal concentration of sodium hypochlorite for root canal preparation ${ }^{33}$. Higher concentrations of sodium hypochlorite are more cytotoxic but have greater tissue dissolution capacity ${ }^{34}$. However, solutions of $5.25 \%$ sodium hypochlorite have been associated with lower postoperative pain. In the first $72 \mathrm{~h}$, lower postoperative pain was observed after single-visit root canal treatment compared to $2.5 \%$ sodium hypochlorite in teeth with irreversible pulpitis ${ }^{33}$ and $1.3 \%$ sodium hypochlorite in necrotic pulps ${ }^{35}$. Studies ${ }^{11,15,16,21}$ that used concentration of sodium hypochlorite (2.5\%) exhibited a significant reduction in postoperative pain after occlusal reduction. The sodium hypochlorite concentration used in the selected studies did not influence pain after endodontic treatment with occlusal reduction.

The determination of working length was mostly done using an apex locator followed by periapical radiography $5,9,11,15,21,23,24$. Tuncer and $\mathrm{Gerek}^{36}$ revealed no difference in postoperative pain between working length measurement with electronic apex locator and digital radiography. Furthermore, Arslan et al. ${ }^{8}$ showed that simultaneous working length measurement and root canal preparation reduce postoperative pain, causing less damage to periapical tissues. The working length varied: in the foramen ${ }^{24}$, at $0.5 \mathrm{~mm}$ from the apex ${ }^{11,21}$, and $1 \mathrm{~mm}$ from the apex ${ }^{5,15,16}$. Studies with working length at $0.5 \mathrm{~mm}^{11,21}$ from the apex and $1 \mathrm{~mm}^{5,15,16}$ from the apex demonstrated significant results of lower postoperative pain after occlusal reduction. 
The type of restorations after endodontic treatment varied: hygroscopic materials (Cotosol, Cavit, MDTemp $)^{5,10,11,13,15,21,22}$, glass ionomer ${ }^{24}$, resin composite ${ }^{8,11,16}$ and ceramic crowns ${ }^{11}$. There are no clinical studies of postoperative pain correlating with the type of temporary restorative material used during endodontic treatment sessions.

As to the obturation, Ezpeleta et al. ${ }^{37}$ emphasized that postoperative pain is significantly associated with the obturation technique used during root canal treatment. However, no clinical studies correlate postoperative pain with the active obturation technique used in the included studies: lateral condensation techniques ${ }^{11,21}$ and thermocompaction ${ }^{24}$. Epoxy resin-based cement was mostly used for obturation: AHplus ${ }^{8,24}$ and Adseal ${ }^{11,21}$. No clinical studies on the incidence of postoperative pain with these endodontic cements were found. Furthermore, only two intracanal medications were used: calcium hydroxide pastes ${ }^{5,9,15}$, and endoseptone ${ }^{16}$. Four studies ${ }^{10,11,16,21}$ did not use any medication.

It is essential to highlight that the studies did not clarify how they measured the occlusal surface reduction of 0.5-1 mm, only mentioning the use of carbon paper and high-speed diamond tips. However, Arslan et al. ${ }^{8}$ used a computerized analysis system to evaluate the relative occlusal force and occlusal surface reduction. Accordingly, studies $^{38,39}$ showed that subjective interpretation of articulating paper markings is wildly inaccurate and an ineffective clinical method for determining the relative occlusal force of tooth contacts. Sutter ${ }^{39}$ recommends using T-Scan, an objective method for occlusal analysis that measures the occlusal force's location.

Reducing the occlusal surface of a tooth that has the structure to be restored is an irreversible step. Also, the occlusal reduction can have a pathological repercussion for the stomatognathic system ${ }^{40,41}$. The present review highlights that reducing the occlusal surface does not influence postoperative pain, with moderate quality of evidence. In this sense, with endodontic treatment performed, even partially, there will be a reduction in postoperative pain. Because of the many methodological differences that can influence postoperative pain, only a small number of studies were included. However, most of these studies had a low risk of bias ("Supplementary materials").

Among the limitations of the present study, the following stand out: language restrictions, the small number of articles included, methodological heterogeneity, the adoption of multiple pain scales, and the different followup periods of observation. Future studies should include investigation of occlusal surface reduction before root canal treatment to prevent postoperative pain on teeth with necrotic pulp and apical periodontitis. It is known that periapical lesions represent an increased risk of postoperative pain ${ }^{27}$. Randomized clinical trials on this subject are scarce so far, making it challenging to consolidate clinical protocols that preserve dental structures.

\section{Conclusion}

The occlusal reduction does not interfere with pain after endodontic instrumentation (at $6,12,24$, or $48 \mathrm{~h}$ ) and the obturation (at 6 and $12 \mathrm{~h}$ ). The certainty of evidence within the studies included in this meta-analysis was considered moderate.

Received: 3 December 2020; Accepted: 14 June 2021

Published online: 07 July 2021

\section{References}

1. Siqueira, J. F. Reaction of periradicular tissues to root canal treatment: Benefits and drawbacks. Endod. Top. 10, 123-147 (2005).

2. Sathorn, C., Parashos, P. \& Messer, H. The prevalence of postoperative pain and flare-up in single- and multiple-visit endodontic treatment: A systematic review. Int. Endod. J. 41, 91-99 (2008).

3. Newton, C. W., Hoen, M. M., Goodis, H. E., Johnson, B. R. \& McClanahan, S. B. Identify and determine the metrics, hierarchy, and predictive value of all the parameters and/or methods used during endodontic diagnosis. J. Endod. 35, 1635-1644 (2009).

4. Owatz, C. B. et al. The incidence of mechanical allodynia in patients with irreversible pulpitis. J. Endod. 33, 552-556 (2007).

5. Parirokh, M. et al. Effect of occlusal reduction on postoperative pain in teeth with irreversible pulpitis and mild tenderness to percussion. J. Endod. 39, 1-5 (2013).

6. Sedgley, C. M. \& Messer, H. H. Are endodontically treated teeth more brittle?. J. Endod. 18, 332-335 (1992).

7. Cohen, S. \& Burns, R.C. Endodontic emergencies-Pathways of the pulp. ed 2. St. Louis, C. V. Mosby Co (1980).

8. Arslan, H., Seckin, F., Kurklu, D. \& Karatas, E. The effect of various occlusal reduction levels on postoperative pain in teeth with symptomatic apical periodontitis using computerized analysis: A prospective, randomized, double-blind study. Clin. Oral Investig. Epub - Apr 30 (2016).

9. Shama, A., Fatima, F. \& Ali, A. Occlusal reduction reduces postoperative pain after endodontic instrumentation. Pakistan Oral Dent. J. 34, 539-542 (2014).

10. Creech, J. L., Walton, R. E. \& Kaltenbach, R. Effect of occlusal relief on endodontic pain. J. Am. Dent. Assoc. 109, 64-67 (1984).

11. Emara, R. S., Abou El Nasr, H. M. \& El Boghdadi, R. M. Evaluation of postoperative pain intensity following occlusal reduction in teeth associated with symptomatic irreversible pulpitis and symptomatic apical periodontitis: A randomized clinical study. Int. Endod. J. 52, 288-296 (2019).

12. Jostes, J. L. \& Holland, G. R. The effect of occlusal reduction after canal preparation on patient comfort. J. Endod. 10, 34-37 (1984).

13. Rosenberg, P. A., Babick, P. J., Schertzer, L. \& Leung, A. The effect of occlusal reduction on pain after endodontic instrumentation. J. Endod. 24, 492-496 (1998).

14. Saatchi, M., P. M. A clinical study of the effect of occlusal reduction on pain after endodontic instrumentation. J. Isfahan Dent. Sch. 1(3\&4), 15-18 (2006).

15. Sheikh, H., Ahmed, M. A. \& Jouhar, R. C. Z. Comparison of post instrumentation pain in teeth during root canal treatment with or with out occlusal reduction. Pakistan Oral Dent. J. 35, 669-701 (2015).

16. Zeidan, B. M. Evaluation the effect of occlusal reduction on postoperative pain in teeth with irreversible pulpitis and mild tenderness to percussion. J. Al Rafidain Univ. Coll. 38, 128-140 (2016).

17. Nixdorf, D. R. et al. Frequency, impact, and predictors of persistent pain after root canal treatment: A National Dental PBRN Study. Pain 157, 159-165 (2016).

18. Higgins, J. P. T. et al. The Cochrane Collaboration' s tool for assessing risk of bias in randomised trials. BMJ. 343, d5928 (2011). 
19. Moher, D., Liberati, A., Tetzlaff, J., Altman, D. G. \& Grp, P. Preferred reporting items for systematic reviews and meta-analyses: The PRISMA statement (reprinted from annals of internal medicine). Phys. Ther. 89, 873-880 (2009).

20. Schünemann, H. et al. GRADE handbook for 3 grading quality of evidence and strength of recommendations. The GRADE Handbook (2013).

21. Ahmed, Y. E. et al. Post-treatment endodontic pain following occlusal reduction in mandibular posterior teeth with symptomatic irreversible pulpitis and sensitivity to percussion: A single-centre randomized controlled trial. Int. Endod. J. 53, 1170-1180 (2020).

22. Zaman, H., Ahmed, S. S. Effect of occlusal reduction on post instrumentation pain in patients with acute irreversible pulpitis. Pakistan Oral Dent. J. 36 (1), 119-121 (2016).

23. Raza, I. et al. Effect of occlusal reduction on frequency of post operative pain relief, following an endodontic instrumentation. Pakistan Oral Dent. J. 36(4), 650-653 (2016).

24. Vianna, E. C. B. et al. Effect of occlusal adjustment on postoperative pain after root canal treatment: A randomized clinical trial. Braz. Dent. J. 31, 353-359 (2020).

25. Arias, A., de la Macorra, J. C., Hidalgo, J. J. \& Azabal, M. Predictive models of pain following root canal treatment: A prospective clinical study. Int. Endod. J. 46, 784-793 (2013).

26. Alí, A. et al. Influence of preoperative pain intensity on postoperative pain after root canal treatment: A prospective clinical study. J. Dent. 45, 39-42 (2016).

27. Siqueira, J. F. et al. Incidence of postoperative pain after intracanal procedures based on an antimicrobial strategy. J. Endod. 28, $457-460$ (2002).

28. Atav Ates, A., Dumani, A., Yoldas, O. \& Unal, I. Post-obturation pain following the use of carrier-based system with AH Plus or iRoot SP sealers: A randomized controlled clinical trial. Clin. Oral Investig. 23, 3053-3061 (2019).

29. Çiçek, E., Koçak, M. M., Koçak, S., Sağlam, B. C. \& Türker, S. A. Postoperative pain intensity after using different instrumentation techniques: A randomized clinical study. J. Appl. Oral Sci. 25, 20-26 (2017).

30. Kherlakian, D. et al. Comparison of the incidence of postoperative pain after using 2 reciprocating systems and a continuous rotary system: A prospective randomized clinical trial. J. Endod. 42, 171-176 (2016).

31. Comparin, D. et al. Postoperative pain after endodontic retreatment using rotary or reciprocating instruments: A randomized clinical trial. J. Endod. 43, 1084-1088 (2017).

32. Sun, C. et al. Pain after root canal treatment with different instruments: A systematic review and meta-analysis. Oral Dis. 24, 908-919 (2018).

33. Farzaneh, S., Parirokh, M., Nakhaee, N. \& Abbott, P. V. Effect of two different concentrations of sodium hypochlorite on postoperative pain following single-visit root canal treatment: A triple-blind randomized. Int. Endod. J. 51, e2-e11 (2018).

34. Gonçalves, L. S., Rodrigues, R. C. V., Andrade Junior, C. V., Soares, R. G. \& Vettore, M. V. The effect of sodium hypochlorite and chlorhexidine as irrigant solutions for root canal disinfection: A systematic review of clinical trials. J. Endod. 42, 527-532 (2016).

35. Mostafa, M. E. H. A. A. et al. Postoperative pain following endodontic irrigation using $1.3 \%$ versus $5.25 \%$ sodium hypochlorite in mandibular molars with necrotic pulps: A randomized double-blind clinical trial. Int. Endod. J. 53, 154-166 (2020).

36. Kara Tuncer, A. \& Gerek, M. Effect of working length measurement by electronic apex locator or digital radiography on postoperative pain: A randomized clinical trial. J. Endod. 40, 38-41 (2014).

37. Alonso-Ezpeleta, L. O. et al. Postoperative pain after one-visit root-canal treatment on teeth with vital pulps: Comparison of three different obturation techniques. Med. Oral Patol. Oral Cir. Bucal. 17, e721-e727 (2012).

38. Kerstein, R. B. \& Radke, J. Clinician accuracy when subjectively interpreting articulating paper markings. Cranio 32, 13-23 (2013).

39. Sutter, B. A. A digital poll of dentists testing the accuracy of paper mark subjective interpretation. Cranio 36, 396-403 (2018).

40. Li, Z., Yang, Z., Lan, T., Zhao, H. \& Liu, Y. Worn is born: The role of the maxillo-mandibular relation in management of worn dentition. Med. Hypotheses 104, 156-159 (2017).

41. Manfredini, D., Castroflorio, T., Perinetti, G. \& Guarda-Nardini, L. Dental occlusion, body posture and temporomandibular disorders: Where we are now and where we are heading for. J. Oral Rehabil. 39, 463-471 (2012).

\section{Acknowledgements}

We acknowledge the support offered by CAPES in postgraduate studies.

\section{Author contributions}

N.C.C.A.: revised the project, performed the electronic search, selected studies, analyzed the methodological quality, and wrote the manuscript. S.R.M.V.: interpretation of data, analyzed the methodological quality, performed the meta-analysis. S.A.S.: revised the project, wrote the manuscript. A.C.A.: revised the project, performed the electronic search, selected studies, and analyzed the methodological quality. C.T.V.T.: revised the project and wrote the manuscript. K.R.: article translation to English and textual corrections. G.Q.M.M.: co-supervisor, manuscript correction, and revision. D.S.A.: supervisor, manuscript correction, and revision.

\section{Funding}

This study was financed in part by the Coordenação de Aperfeiçoamento de Pessoal de Nível Superior-Brazil (CAPES)-Finance Code 001.

\section{Competing interests}

The authors declare no competing interests.

\section{Additional information}

Supplementary Information The online version contains supplementary material available at https://doi.org/ 10.1038/s41598-021-93119-6.

Correspondence and requests for materials should be addressed to G.Q.M.M.

Reprints and permissions information is available at www.nature.com/reprints.

Publisher's note Springer Nature remains neutral with regard to jurisdictional claims in published maps and institutional affiliations. 
(c) (i) Open Access This article is licensed under a Creative Commons Attribution 4.0 International cc) License, which permits use, sharing, adaptation, distribution and reproduction in any medium or format, as long as you give appropriate credit to the original author(s) and the source, provide a link to the Creative Commons licence, and indicate if changes were made. The images or other third party material in this article are included in the article's Creative Commons licence, unless indicated otherwise in a credit line to the material. If material is not included in the article's Creative Commons licence and your intended use is not permitted by statutory regulation or exceeds the permitted use, you will need to obtain permission directly from the copyright holder. To view a copy of this licence, visit http://creativecommons.org/licenses/by/4.0/.

(C) The Author(s) 2021 